\title{
Combined pulmonary lobectomy for surgical treatment of a malignant fibrous histiocytoma of the chest wall: a case report
}

\author{
Yi Liu, Gang Chen*, Yi Wu, Renwang Liu, Song Xu, Jun Chen ${ }^{*}$ and Qinghua Zhou*
}

\begin{abstract}
Background: A malignant fibrous histiocytoma (MFH) rarely originates from the chest wall.

Clinical findings: In this case, we describe a 59-year-old Chinese woman who presented with an enormous mass originating from the left chest wall and involving the left upper pulmonary lobe.

Therapy: After a radical en-block resection of the entire chest mass with left upper pulmonary lobectomy, and the chest wall reconstruction, a histopathologic diagnosis of the giant cell MFH was rendered. She has done well postoperatively, showing no local recurrence or distal disease in an 8-month follow-up period.

Conclusion: Although a MFH originating from the chest wall is rare, it should be considered in the differential diagnosis of a chest wall tumor.

Virtual slides: The virtual slide(s) for this article can be found here: http://www.diagnosticpathology.diagnomx.eu/ vs/8895569301129379
\end{abstract}

Keywords: Malignant fibrous histiocytoma, Differential diagnosis, Chest wall tumor

\section{Introduction}

Malignant fibrous histiocytoma (MFH) is a common soft tissue sarcoma, which is often found in adults with primary location of the extremities or retroperitoneum. However, MFH rarely originates from the chest wall [1]. Here we report the case in a 59-year old woman with a left chest big mass involving the left upper pulmonary lobe that was initially suspicious of lung carcinoma invading the chest wall. Ultimately, the chest wall giant cell malignant fibrous histiocytoma was confirmed following en-block resection of chest wall mass and left upper lobectomy.

\section{Case presentation}

A 59-year-old Chinese woman was admitted to the hospital with the chief complaint of a progressively enlarging mass in the left thoracic wall over the past seven months. At the initial examination, the tumor slightly

\footnotetext{
*Correspondence: chengang450@163.com; huntercj2004@yahoo.com; zhough135@163.com

Department of Lung Cancer Surgery, Tianjin Lung Cancer Institute, Tianjin Medical University General Hospital, Tianjin 30052, China
}

protruded from the chest wall with the $2 \mathrm{~cm}$ in diameter, and gradually grew over in the past several months. In one month ago, she underwent a percutaneous transthoracic needle biopsy of the mass in another hospital. Following the puncture, the tumor promptly increased significantly in size, impacting blood flow to the left arm. On this admission, we conducted a physical examination and palpated the soft painless left lateral chest wall large mass with the $9 \mathrm{~cm}$ in diameter. She denied smoking history and had no family history of lung cancer. A review of her systems was noncontributory. The results of a peripheral blood count, baseline serum chemistry screening, and urinalysis were normal on admission, as were tumor biomarker tests and a purified protein derivative test for tuberculosis.

Enhanced chest computed tomography (CT) with threedimensional reconstruction of the ribs showed an enormous soft tissue mass in the left chest wall (Figure 1), which extended into the left lung and mediastinum, exerted pressure on the heart, skewing it to the right, and had eroded the middle portion of the fifth rib. The density of the tissue mass was uneven and a necrotic area was 


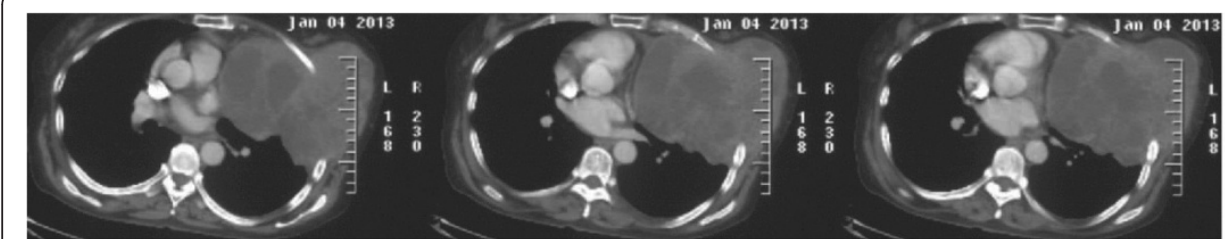

A
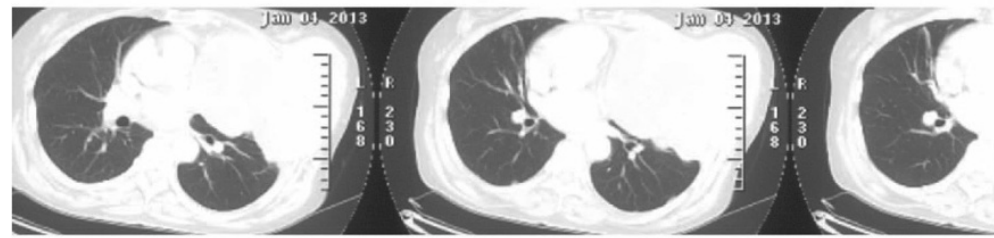

B

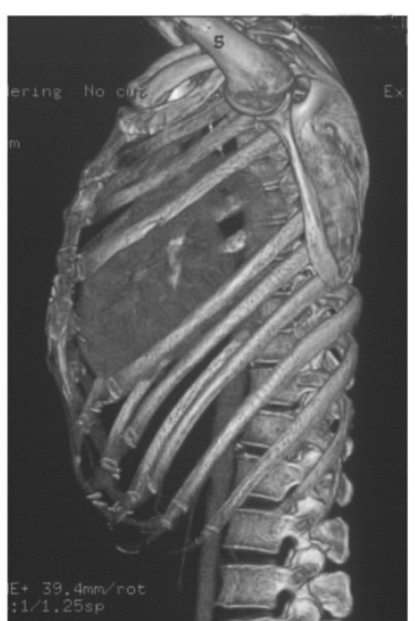

C

Figure 1 Enhanced chest CT scan before operation. A: In the mediastinal window, the CT scan revealed the tumor encroaching on the superior vena cava (right panel), surrounding the right upper lobe bronchus (middle panel), and invading the right pulmonary artery (left panel). B: In the lung window. C: In the mediastinal window, coronal.

visible. The maximum cross-sectional diameter of the tumor mass was $13.3 \times 15.7 \mathrm{~cm}^{2}$. Moreover, an ECT bone scan also demonstrated the destruction of the middle of the fifth rib. In additional, a CT scan of her abdomen and magnetic resonance imaging of her brain were all normal. Her bronchial tree also appeared normal on bronchoscopic examination, with no indication of malignancy in the biopsy and washings procured.

After anesthesia and a double-lumen intubation had been established, a left posterolateral incision was performed. The chest wall tumor invaded the left upper pulmonary lobe as well as the fourth, fifth, and sixth ribs. The tumor had eroded the middle portion of the fifth rib; the heart was impacted by the tumor and mediastinal pleural adhesions were present. We freed-up the outer margin of the tumor and excised the fourth, fifth, and sixth ribs. Intercostal tissue was included with a margin over $5 \mathrm{~cm}$. We extracted the tumor and the invaded left upper lobe of lung to remove the pressure on the heart. We then performed the left upper pulmonary lobectomy. Finally, we joined four $10 \times 10 \mathrm{~cm}^{2}$ polyester surgical patches (knitted type) into one $20 \times 20 \mathrm{~cm}^{2}$ patch and this patch was sutured to the chest wall defect for chest wall reconstruction.

The neoplasm, which was removed by surgical resection, weighed $2.080 \mathrm{~kg}$ and was $20 \mathrm{~cm} \times 18 \mathrm{~cm} \times 9 \mathrm{~cm}$ in size, as shown in Figure 2A. There were multiple nodules on its surface and a cross section of the tumor was honeycombed in appearance. The pathology report described a giant cell malignant fibrous histiocytoma cell tumor (Figure 2B), with the immunohistochemistry of CD68 (+), Vimentin (+), SMA, CK and S-100 (-) (Figure $2 \mathrm{C}$ ), invading the left upper lung lobe and the

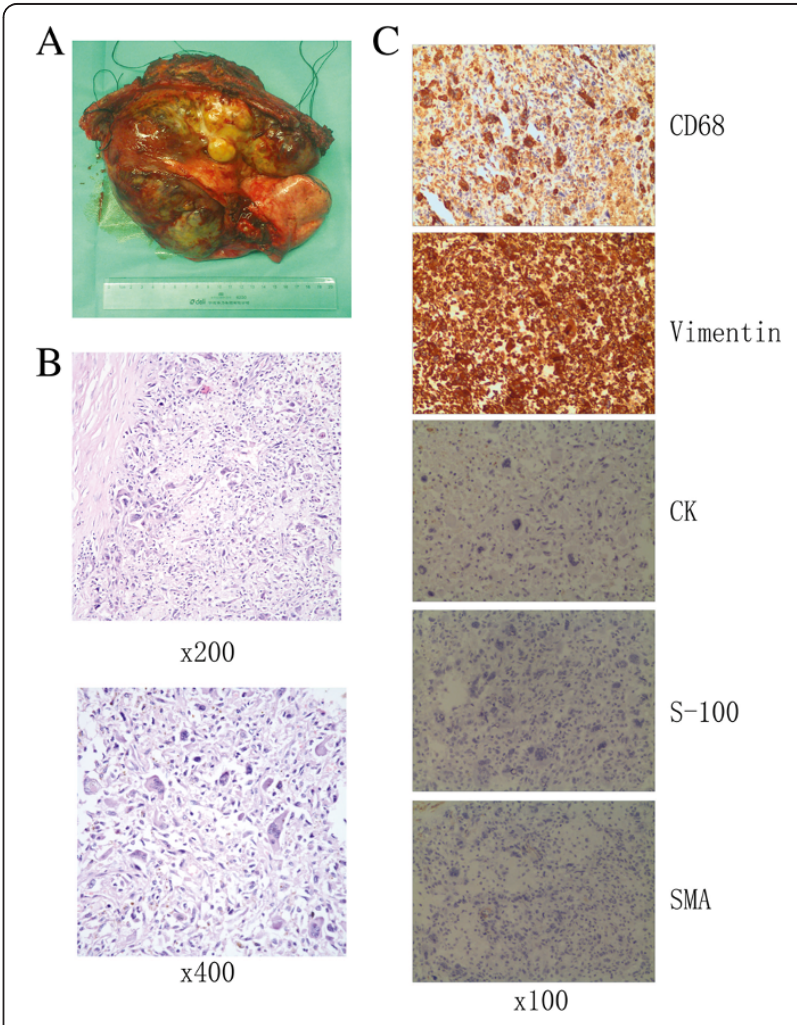

Figure 2 Histopathological images. (A) Gross features of the $20 \mathrm{~cm}$ mass with the invaded fifth rib and the left upper pulmonary lobe; (B) Hematoxylin and eosin (H\&E) staining of a giant cell malignant fibrous histiocytoma cell tumor. (C) Immunohistochemical staining of primary tumor with antibodies to CD68 (+), Vimentin (+), CK (-), S-100 (-) and SMA (-). 
middle of the fourth, fifth, sixth ribs, with no metastases to the mediastinal lymph nodes. Her postoperative course was uneventful. She was discharged 12 days after surgery and showed no signs of local recurrence or distal disease at an 8-month follow-up visit (Figure 3).

\section{Discussion}

A MFH is the most common soft tissue tumor found in adults. It is primarily located in the extremities or retroperitoneum; however, it rarely appears on the chest wall. A MFH was first reported by O'Brien and Stout in 1964 [2]. They described it as "malignant fibrous xanthoma". The tumor preferentially involves the deep fascia, skeletal muscles, or superficial subcutaneous tissue. A MFH has a high propensity for local recurrence and distant metastases. Weiss and Enzinger [3] reported that a local recurrence rate of $44 \%$ and metastatic recurrence rate of $42 \%$ for MFHs. Several MFH subtypes have been described in the literature: storiform-pleomorphic, giant cell, inflammatory, and myxoid [4]. The mean survival of patients without surgery is 11.7 months; it is 23.2 months for patients who undergo surgery [5]. MFHs have been reported to occur with increasing frequency in patients treated with radiotherapy for other malignant diseases; however, no prior exposure to ionizing radiation or history of malignancy was documented in our case. The role of either chemotherapy or radiotherapy as primary or adjuvant treatment for MFHs is currently unclear. Favorable factors for MFHs are: UICC/AJCC stage I and II, superficial location, myxoid type, and patient under 50 years of age. Under these conditions, the rational for radical enblock resection of the tumor is supported [6-10].

$\mathrm{CT}$ and magnetic resonance imaging are useful for the radiological evaluation of the soft tissue component. According to one report, the imaging features of MFHs in the chest wall are nonspecific and only the myxoid type showed a high-intensity pattern with T1 weighed images (T1WI) and T2 weighted images (T2WI). The mass usually shows intense enhancement on CT with a clear margin separating it from the surrounding tissue [11].

For a chest wall MFH, wide resection is the first treatment choice; chemotherapy should also be considered. King reported the relationship between the distance of the lateral margin of the excision from the tumor and the recurrence rate. They found a distance of $4 \mathrm{~cm}$ was associated with a recurrence rate of $44 \%$; whereas a distance of $2 \mathrm{~cm}$ was associated with a $71 \%$ recurrence rate [12]. They recommended an excisional distance of $4 \mathrm{~cm}$. Naoya Yoshida et al. reviewed $39 \mathrm{MFH}$ cases and found that patients with a negative surgical margin were alive without recurrence, irrespective of the surgical margin distance [12].

After radical en-block resection, the chest wall should be reconstructed. Classically, various flaps such as a major pectoral muscle flap, a major pectoral myocutaneous flap, or a latissimus dorsi myocutaneous flap and pedicled omentum have been utilized for reconstruction. Currently, reconstruction with Marlex mesh is widely used. Several studies have reported that a chest wall reconstruction with Marlex mesh is successful and inexpensive. In this case, the chest wall defect was too large for reconstruction with a flap. We performed the reconstruction with a polyester surgical patch (knitted type), and we joined four $10 \times 10 \mathrm{~cm}^{2}$ patches into one $20 \times 20 \mathrm{~cm}^{2}$ patch to reconstruct the chest wall. Postoperative complications such as skin dehiscence, skin necrosis, and infection did not occur. We found that reconstruction of the chest wall with a polyester surgical patch for a large defect is safe, rapid, and simple. We consider that radical enblock resection for an enormous chest wall $\mathrm{MFH}$ and

A

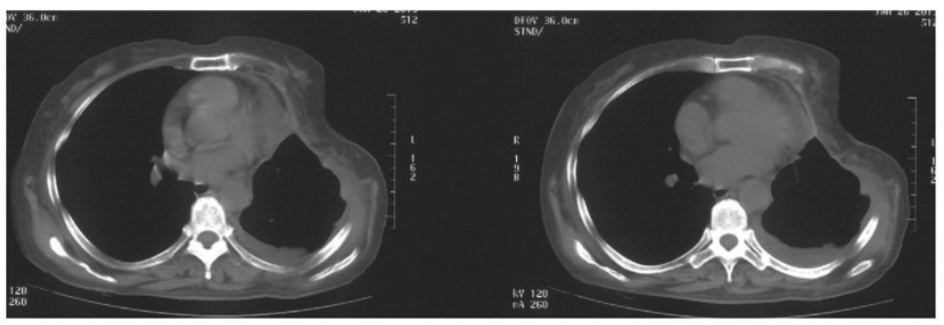

B

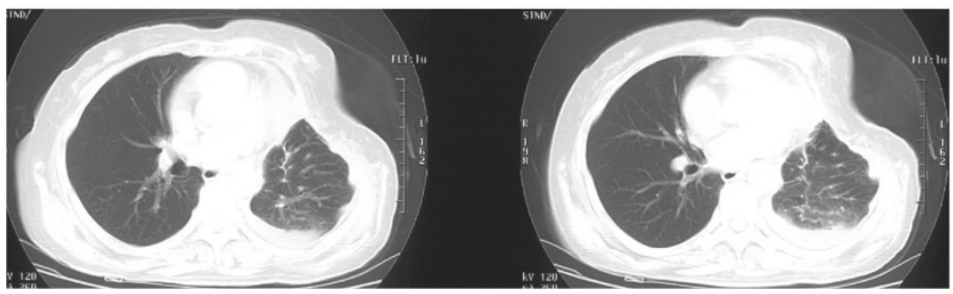

Figure $\mathbf{3}$ CT scans after operation. The chest CT scan was performed in 7 days after the operation. $\mathbf{A}: \operatorname{In}$ the mediastinal window; B: In the lung window. 
reconstruction is a safe procedure that may increase longterm survival.

In differential diagnosis of a chest wall tumor, malignant fibrous histiocytoma should be seriousely considered.

\section{Conclusion}

Malignant fibrous histiocytoma be considered in the differential diagnosis of a chest wall tumor.

\section{Ethical approval}

Our research has been approved by the ethical committee of Tianjin Medical University General Hospital.

\section{Consent}

The patient granted written informed consent for publication of this manuscript and the accompanying images. A copy of the written consent is available for review by the Editor-in-Chief of this journal.

\section{Competing interests}

The authors declare that they have no competing interests.

\section{Authors' contributions}

YL collected all data and authored the manuscript. GC, YW, RWL and SX were responsible for patient care and analysis of follow-up data. QZ participated in data analysis and manuscript revisions. JC and QHZ performed the surgical procedure, also contributing to data analysis and shaping of the manuscript. All authors have read and approved the final manuscript.

\section{Acknowledgements}

This work was supported in part by grants from the Science and Technology Support Key Program of Tianjin (12ZCDZSY16100) and the Ministry of Education for New Century Excellent Talents (NCET-10-0956). Funding sources had no role in study design, data collection, and analysis; in decision to publish; or in preparation of the manuscript.

Received: 11 November 2013 Accepted: 4 December 2013

Published: 23 January 2014

\section{References}

1. Hurtado-Cordovi J, Avezbakiyev B, Frieri M, Freedman L, Gebre W: Cutaneous inflammatory malignant fibrous histiocytoma presenting with a leukemoidreaction: a case report and review of the literature. Case Rep Med 2012, 2012:798629.

2. Chou YS, Liu CY, Chen WM, et al: Follow-up after primary treatment of soft tissue sarcoma of extremities: impactof frequency of follow-up imaging on disease-specific survival. J Surg Oncol 2012, 106(2):155-161.

3. Suzuki S, Watanabe S, Kato H, Inagaki H, Hattori H, Morita A: A case of cutaneous malignant fibrous histiocytoma with multiple organmetastases. Kaohsiung J Med Sci 2013, 29(2):111-115.

4. Wang LL, Xie H, Fu HL, et al: An adolescent presenting with malignant fibrous histiocytoma of the testis: a case report. J Med Case Rep 2013, 7(1):30.

5. Hollmig ST, Kirkland EB, Henderson MT, Tang JY, Gladstone HB: The evolving conception and management challenges of malignant fibroushistiocytoma. Dermatol Surg 2012, 38(12):1922-1929.

6. Jeon YH, Park KS: Successful management of a recurrent primary of the lung: report of a case. Korean J Thorac Cardiovasc Surg 2012, 45(5):345-347.

7. Li J, Yin WH, Takeuchi K, Guan H, Huang YH, Chan JKC: Inflammatory myofibroblastic tumor with RANBP2 and ALK gene rearrangement: a report of two cases and literature review. Diagn Pathol 2013, 8:147.

8. Lin XY, Wang Y, Liu Y, Sun Y, Miao Y, Zhang Y, et al: Pulmonary sclerosing hemangioma presenting with dense spindle stroma cells: a potential diagnostic pitfall. Diagn Pathol 2012, 7:155.

9. Atoini1 F, Ouarssani A, Hachimi MA, Aitthou F, Guenoun FZ, Elmejereb C, et al: Diffuse large B-cell lymphoma presenting as large anterior chest wall mass involving pleura and lung: a possible result of post-traumatic chronic inflammation. Thoracic Cancer 2012, 3(1):79-83.

10. Tacconi F, Ambrogi $V$, Mineo D, Mineo TC: The impact on quality of life after en-bloc resection for non-small-cell lung cancer involving the chest wall. Thoracic Cancer 2012, 3(4):326-333.

11. Lee HJ, Yang HM, Choi YS, et al: A Therapeutic strategy for metastatic malignant fibrous histiocytoma ThroughMesenchymal stromal cell-mediated TRAIL production. Ann Surg 2012, 257(5):952-960.

12. Yoshida N, Miyanari N, Yamamoto Y, Egami H: Successful treatment of malignant fibrous histiocytoma originating in the chestwall: report of a case. Surg Today 2006, 36(8):714-721.

doi:10.1186/1746-1596-9-21

Cite this article as: Liu et al: Combined pulmonary lobectomy for surgical treatment of a malignant fibrous histiocytoma of the chest wall: a case report. Diagnostic Pathology 2014 9:21.

\section{Submit your next manuscript to BioMed Central and take full advantage of:}

- Convenient online submission

- Thorough peer review

- No space constraints or color figure charges

- Immediate publication on acceptance

- Inclusion in PubMed, CAS, Scopus and Google Scholar

- Research which is freely available for redistribution 